\title{
IGRF candidate models at times of rapid changes in core field acceleration
}

\author{
Erwan Thébault ${ }^{1}$, Arnaud Chulliat ${ }^{1}$, Stefan Maus ${ }^{2}$, Gauthier Hulot ${ }^{1}$, Benoit Langlais $^{3}$, \\ Aude Chambodut ${ }^{4}$, and Michel Menvielle ${ }^{5}$ \\ ${ }^{1}$ Équipe de géomagnétisme, Institut de Physique du Globe de Paris, UMR 7154, CNRS-INSU, Univ. Paris Diderot, Paris, France \\ ${ }^{2}$ National Geophysical Data Center, NOAA E/GC1, 325 Broadway, Boulder, CO 80305, USA \\ ${ }^{3}$ Laboratoire de Planétologie et Géodynamique de Nantes, UMR 6112, CNRS, Univ. de Nantes, Nantes, France \\ ${ }^{4}$ Institut de Physique du Globe de Strasbourg, UMR 7516, Université de Strasbourg, Strasbourg, France \\ ${ }^{5}$ Laboratoire Atmosphères, Milieux, Observations Spatiales (UMR 8190-CNRS), Paris, France
}

(Received December 25, 2009; Revised May 10, 2010; Accepted May 17, 2010; Online published December 31, 2010)

\begin{abstract}
We submit three candidate models following the call for IGRF-11. We apply a simple modeling approach in spherical harmonics based on a quadratic Taylor expansion for the internal field time variations. We use the $D_{\text {st }}$ magnetic index as a proxy for the external field variations. In order to compensate for the limitations incurred by such a conventional approach, we focus on the optimal selection of satellite data in space and time. We also show that some a priori knowledge about the core field state helps us to avoid the pitfall encountered in the case of rapid changes of core field accelerations. Indeed, various acceleration events of relevance for the IGRF 11 th occurred between 2003 and 2010, one of them being a geomagnetic jerk. They could entail disagreements between IGRF candidate models for the secular variation (SV) if data prior to 2008 are used. Our SV and main field (MF) candidate models have a root mean square uncertainty less than $6 \mathrm{nT} / \mathrm{yr}$ and $8 \mathrm{nT}$, respectively, with respect to the modeled magnetic field contributions. These values correspond to the intrinsic error associated with truncating SV and MF models to spherical harmonic degree 8 and 13, respectively, as requested for IGRF models.
\end{abstract}

Key words: Magnetic field, main field, secular variation, modeling, IGRF, temporal extrapolation, core field acceleration.

\section{Introduction}

The geomagnetic field at the Earth's surface is by far dominated by the field from the Earth's core. Representing this dominant magnetic field and forecasting it to the near future has a large variety of practical and scientific applications, from aeronautic navigation to geophysical exploration and core field dynamic studies. For instance, marine, aeromagnetic and ground magnetic surveys, widely used in geophysical mapping of the Earth's crust, require the prior subtraction of standard main field models (Hamoudi et al., 2007; Hemant et al., 2007; Korhonen et al., 2007; Maus et al., 2007).

Every five years, the International Association of Geomagnetism and Aeronomy (IAGA) releases the International Geomagnetic Reference Field (IGRF), a standard model of the main field (MF) and its predicted secular variation (SV) for the following 5 years. Both models are given as spherical harmonic ( $\mathrm{SH}$ ) expansions. In addition, the IAGA working group in charge of publishing the IGRF (V-MOD) retrospectively revises the previous IGRF model into a definitive geomagnetic reference model describing the field up to the end of the previous epoch.

Here, we describe three candidate models that we pro-

Copyright (C) The Society of Geomagnetism and Earth, Planetary and Space Sciences (SGEPSS); The Seismological Society of Japan; The Volcanological Society of Japan; The Geodetic Society of Japan; The Japanese Society for Planetary Sciences; TERRAPUB.

doi:10.5047/eps.2010.05.004 posed for the IGRF 11th generation (IGRF-11). We derived two MF models respectively centered on epochs 2005.0 and 2010.0, and one predictive SV model covering the period from 2010.0 to 2015.0. These models were produced and submitted by the Institut de Physique du Globe de Paris (IPGP) in collaboration with four groups. The candidate models benefited from the availability of more than 10 years of magnetic measurements from the Ørsted (Olsen et al., 2000) and CHAMP (Reigber et al., 2002) satellites. They also relied on the continuous series of magnetic measurements provided by ground magnetic observatories.

However, because of the well-known problems raised by their inhomogeneous spatial distribution, we did not directly use observatory data in our modeling procedure beyond the purpose of selecting, correcting data, and a posteriori assessing the quality of our IGRF-11 candidates at the Earth's surface. Likewise, Ørsted data were only used for the validation of models at satellite altitude.

In this paper, we report on the steps we followed to generate the candidate models. We provide the information requested by the IGRF-11 call for candidates (Maus and Finlay, internal IAGA communication). We also introduce an original evaluation scheme based on quasi-definitive and definitive observatory data.

\section{Data Selection}

A major difficulty in geomagnetic field modeling is to account for rapidly time-varying external fields that are difficult to parameterize with mathematical models. A com- 
mon approach is to try to minimize these contributions by a combination of data selection and pre-processing (see e.g. Thomson and Lesur, 2007 for a discussion). Here, we use version-50 CHAMP level 2 data up to 12th August 2009. The CHAMP scalar and vector data were sub-sampled every 20 seconds, corresponding to about $150 \mathrm{~km}$ along-track spacing. External field signals are particularly difficult to represent near and in the polar areas. For the scalar data, separate sets were thus compiled for mid latitudes $\left(-60^{\circ}\right.$ to $60^{\circ}$ magnetic latitudes) and polar regions $\left(>50^{\circ}\right.$ and $\left.<-50^{\circ}\right)$. Both scalar and vector data were used at mid latitudes but selected for 22:00-5:00 local time in order to minimize the contributions from the ionospheric $S_{\mathrm{q}}$ field. In contrast, only scalar data were used in the polar regions and at all local times, as vector data at these latitudes may be contaminated by magnetic fields associated with fieldaligned currents. In all cases, the attitude of the dual-head star camera was requested to be in operating mode.

Data selections based on the $D_{\text {st }}$ and $a_{\mathrm{m}}$ indices (see e.g., Menvielle and Marchaudon, 2006) were also applied. The $D_{\text {st }}$ index, which measures the activity of the magnetic field generated by the ring current, was requested to never exceed $\pm 30 \mathrm{nT}$. In addition, in polar regions the maximum value of the $D_{\text {st }}$ time derivative was set to $5 \mathrm{nT} / \mathrm{h}$. Since other magnetospheric sources may occur, the $a_{\mathrm{m}}$ index, which measures the general magnetic activity at the planetary scale, was requested to satisfy $a_{\mathrm{m}} \leq 27$ after having met the same requirement over the previous three hours. At mid latitudes, the maximum value of the $D_{\text {st }}$ time derivative was set to $2 \mathrm{nT} / \mathrm{h}$ and the $a_{\mathrm{m}}$ was requested to satisfy $a_{\mathrm{m}} \leq 12$ following three hours where $a_{\mathrm{m}} \leq 15$. In all cases, the Interplanetary Magnetic Field (IMF), related to the solar wind, had to be within $\pm 8 \mathrm{nT}$ for the $Y$ component and between $-2 \mathrm{nT}$ and $6 \mathrm{nT}$ for the $Z$ component.

Finally, the along-track residual mean square (rms) between the data and the POMME-5 model predictions (http://www.geomag.us/models/pomme5.html) were sorted by longitude and by date separately for mid latitude, north polar and south polar tracks. Then, a track was considered "noisy", and thus rejected, if its rms value was found to be larger than the rms at neighboring tracks by more than $3 \mathrm{nT}$.

A number of corrections were also applied to the selected data in order to mitigate the effect of other time varying magnetic fields on our internal field candidate models. Estimated angular corrections for the misalignment between the CHAMP satellite magnetometer and the star-tracker reference systems were applied to correct the vector data following the procedure described in Maus et al. (2005a). The magnetic signals of motional induction in the oceans due to the 8 major tidal constituents up to spherical harmonic degree 45, as predicted by Kuvshinov and Olsen (2004), which could reach $3 \mathrm{nT}$ at satellite altitudes, were subtracted. However, we did not apply any correction for the "diamagnetic effect" as proposed by Lühr et al. (2003), as this correction was not available for the latest CHAMP data at the time we derived our models.

\section{Model Parameterization}

We chose a simple parameterization for representing the internal Earth's magnetic field. The internal magnetic field
$B_{\text {int }}$ derives from a scalar potential $V$

$$
V_{\text {int }}(r, \theta, \phi, t)=a \sum_{n=1}^{N} \sum_{m=-n}^{n}\left(\frac{a}{r}\right)^{n+1} g_{n}^{m}(t) Y_{n}^{m}(\theta, \phi)
$$

in such a way that $B_{\text {int }}=-\nabla V_{\text {int }}(r, \theta, \phi) . \theta$ is the geocentric co-latitude, $\phi$ the longitude, $r$ the radius and $a=$ $6371.2 \mathrm{~km}$ the Earth's reference radius. The integers $n$ and $m$ are respectively the degree and order of the Schmidt quasi-normalised spherical harmonic $Y_{n}^{m}(\theta, \phi)$. Gauss coefficients $g_{n}^{m}$ with negative order are usually denoted $h_{n}^{m}$ in geomagnetism.

The time dependence of the internal Gauss coefficients was modeled as a quadratic Taylor expansion around a centre epoch defined by $t_{0}$

$$
g_{n}^{m}(t)=g_{n}^{m}+\left(t-t_{0}\right) \dot{g}_{n}^{m}+0.5\left(t-t_{0}\right)^{2} \ddot{g}_{n}^{m} .
$$

The coefficients $g_{n}^{m}, \dot{g}_{n}^{m}$, and $\ddot{g}_{n}^{m}$, were estimated up to SH degree 15,10 , and 5, respectively. We co-estimated the static external magnetic field up to $\mathrm{SH}$ degree 2 and assumed a temporally varying component of degree 1 parameterized by the $D_{\mathrm{st}}$ index split into its external $E_{\mathrm{st}}$ and internal $I_{\mathrm{st}}$ counterpart (Maus and Weidelt, 2004; Olsen et al., 2005a). The external field was expressed with respect to the colatitude and the longitude of the internal dipole field. The inverse problem was linearized around an initial guess $m_{0}$ and the vectors of coefficients $m$ for epochs 2005.0 and 2009.0 were estimated by means of a least-squares iterative scheme minimizing the cost function (following e.g. Olsen et al., 2000) without explicit spatial or temporal regularization

$$
m_{i+1}=m_{i}+\left(A^{T} W A\right)^{-1} A^{T} W^{T}\left(d-f\left(m_{i}\right)\right),
$$

where $d$ is the data vector, $f\left(m_{i}\right)$ the SH model predictions at iteration $i, W$ the weight matrix, and $A$ the design matrix. We used the definitive IGRF model at epoch 2000 (Macmillan and Maus, 2005) up to SH degree 13 without time dependency as a starting model $m_{0}$. The solution was found to converge in four (resp. five) iterations for a model centered on epoch 2005.0 (resp. 2009.0). We checked with an independent initial model (POMME-5) that the final solution was not tied to this a priori information. The outliers were not removed or down-weighted during the iteration process.

\section{Definitive Candidate Model for 2005.0}

\subsection{Data decimation}

In the first modeling step, we started by deriving many models centered on 2005.0 with various time windows (from $\pm 1 \mathrm{yr}$ to $\pm 2.5 \mathrm{yr}$ ) in order to define the optimal time interval. We found that the mean of residuals between the data and the model predictions increased with the width of the time window. This growing bias likely reflects the fact that such models failed to capture some of the behavior of the internal magnetic field over that specific period. The epoch 2005-2006 is indeed characterized by an episode of intense field acceleration pulse at the core surface (Chulliat et al., 2010) bounded by two geomagnetic jerks, in 2003 (Olsen and Mandea, 2007) and 2007 (Chulliat et al., 2010). 


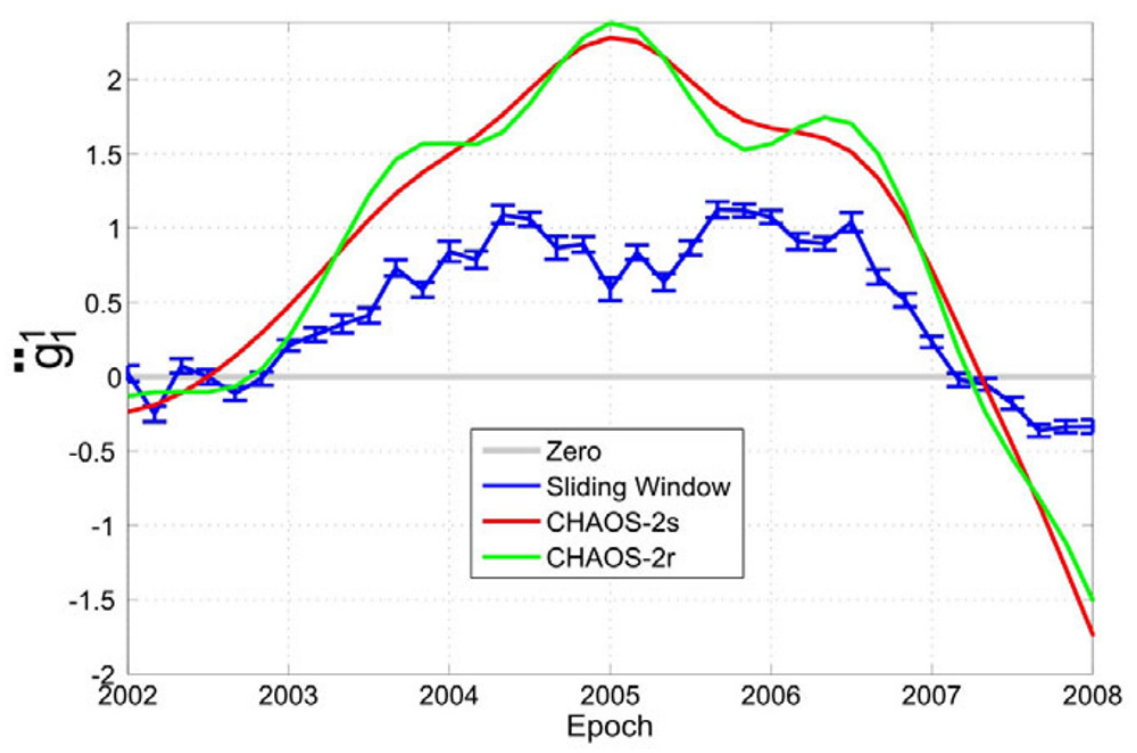

Fig. 1. Acceleration of coefficient $g_{1}^{1}$ with time (in $\mathrm{nT} / \mathrm{yr}^{2}$ ) derived from CHAOS-2s (red) and CHAOS-2r (green) as well as from a modeling technique based on a sliding time window of width $\pm 1.5 \mathrm{yr}$ (see text). The error bars in blue are formal error estimated from the covariance matrix.

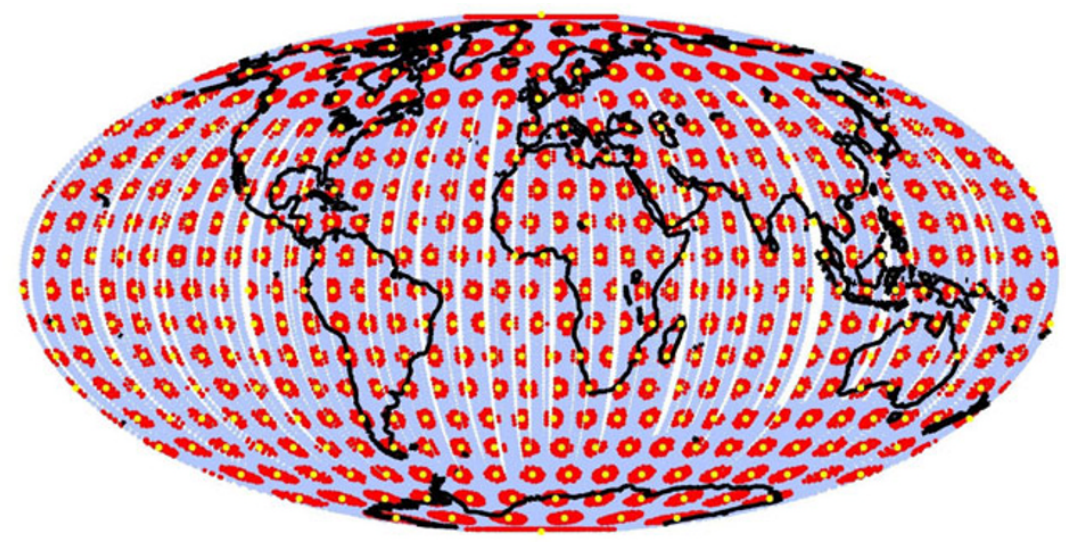

Fig. 2. Selection of the original pre-processed CHAMP intensity data (light blue), belonging to spherical caps (in red) centered on the nodes (yellow dots) of an equal area grid.

We plot in Fig. 1 the time variations of the $\ddot{g}_{1}^{1}$ coefficient as provided by the CHAOS-2r and CHAOS-2s models (' $r$ ' and 's' stand for 'rough' and 'smooth'; Olsen et al., 2009). We chose this model because its 'rough' version is expected to compare best with our non-regularized models, and the coefficient $\ddot{g}_{1}^{1}$ because it clearly contains the signature of the 2005-2006 pulse. The series of acceleration coefficients derived by our technique over a sliding time window of $\pm 1.5 \mathrm{yr}$ shows that quadratic Taylor expansion models may have difficulties recovering the rapid variation of the field around 2005 (assuming that the CHAOS-2 model captures well the internal field time variations).

In order to mitigate this effect, modeling strategies relying on higher time derivatives could have been used. However, we decided to keep with a quadratic Taylor expansion and to look for the best compromise between selecting the optimum data coverage in space and choosing a time interval around the centre epoch within which neglecting the third time derivative of internal Gauss coefficients is accept- able. Our approach involved two steps.

First, for a given width of the temporal window over which the model is meant to be computed (that will be defined below), we iteratively adjusted the bounding epochs in order to have an arithmetic mean epoch equal to 2005.0, within an arbitrary tolerance of $\pm 0.042 \mathrm{yr}$ ( \pm 15 days $)$, and an equal amount of data before and after 2005.0. This procedure is important because the selected CHAMP data are unevenly distributed before and after 2005.0. Neglecting this detail would have led to a model biased towards epochs where more data are available. Note that another option (not retained here) could have been to weigh the data according to their dates.

In a second step, the set of pre-processed CHAMP data selected within the chosen window was further decimated to optimize the geographical distribution. We kept data close to the nodes of a quasi-regular grid that preserves the orthogonal properties of spherical harmonics using a minimum, but sufficient, number of data locations (see Thébault, 


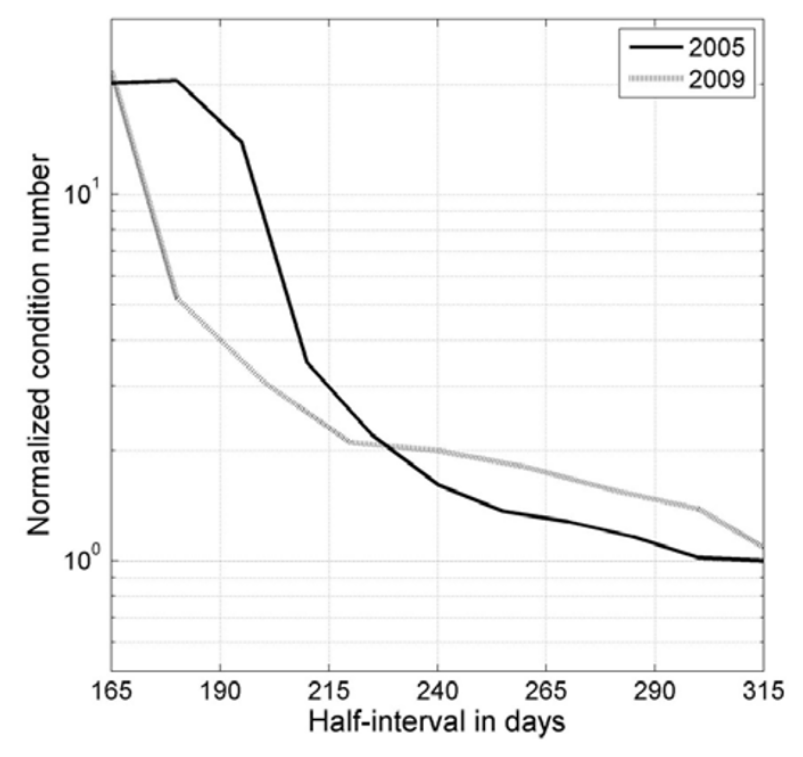

Fig. 3. Plot of the normalized condition number of the inverse matrix as a function of the width of the time window centered on 2005.0 (solid line) and on 2009.0 (dashed line).

2006 for details). Figure 2 illustrates an arguably optimal decimation of the dataset to achieve the purpose of modeling the main field to SH degree 15 . We filled the diagonal weight matrix $W$ for the scalar data in the regions between $50^{\circ}$ and $60^{\circ}$ latitude (North and South). These weights were designed to provide a smooth transition from the lower to the higher latitudes. For instance, mid-latitude weights were linearly tapered from 1 at $50^{\circ}$ to 0 at $60^{\circ}$ geomagnetic latitude. In addition, all vector and scalar data belonging to each of the spherical caps (in red) were weighted proportionally to their distance from the cap's centre, with a weight equal to one at the centre and only a half along the edge. In addition, each data within a cap was weighted according to the number of data within the cap. This procedure is equivalent to an equal area data weighting.

Using this approach, we then looked for the time interval allowing for the best trade-off between a snapshot modeling of the main field and the good conditioning of the inverse problem. We applied an iterative procedure that inverts CHAMP satellite data belonging to progressively narrower time windows. We computed each time the values of the condition number with respect to the inversion. We recall the condition number $C$ is defined as the ratio of extreme eigenvalues $\lambda\left(C=\lambda_{\max } / \lambda_{\min }\right)$ of the inverse matrix $A^{T} W A$. High condition numbers, corresponding to ill-posed inverse problems, were thus avoided. Plotting $C$ as a function of the window width led us to choose the interval \pm 240 days ( \pm 9 months) because below this width the conditioning was found to degrade significantly (Fig. 3). This time interval corresponded to our "optimum" time window for which the model could be considered as robust in the sense that Gauss coefficients are well determined. This optimal time window remains smaller than the duration for which neglecting the third time derivative would raise difficulties (see Fig. 1). Note that this approach requires that the selected and corrected magnetic measure- ments belonging to the time interval have a good signal to noise ratio and are not biased. For shorter time spans, regularization in space and time would have been needed, which we precisely intended to avoid.

\subsection{Assessment of the 2005.0 candidate model}

The amount of scalar and vector data, the root mean square (rms) values and mean values of the residuals are listed in Table 1. In Fig. 4, we compare our parent 2005.0 candidate model truncated to $\mathrm{SH}$ degree 13 with three models: CHAOS-2r, GRIMM-2 (an updated version of GRIMM; Lesur et al., 2008), and POMME-5 (an updated version of POMME-3, see Maus et al., 2006). This is done in the spectral domain by plotting the power spectra of the differences between our candidate 2005.0 and those three models.

The rms difference between our model predictions (calculated with coefficients up to SH 15) at the Earth's surface and CHAOS-2r, GRIMM-2, and POMME-5 models, are $4.4 \mathrm{nT}, 4.7 \mathrm{nT}$, and $4.4 \mathrm{nT}$, respectively. These values compare well with the CHAMP data misfit reported in Table 1. This suggests that all four models can be considered as noisy versions of the same 'true' core field model, assuming of course that none of the models is biased. However, a few intriguing features are worth mentioning. First, Fig. 4 shows that CHAOS-2r, GRIMM-2, and POMME-5 models all deviate from our model in an almost similar fashion, in particular between SH degree 8 and 13. Second, the mean of residuals between the selected CHAMP data and our model predictions displays a small bias, even in nonpolar regions (Table 1). This suggests that our parameterization misses some magnetic field contributions, which in turn could introduce biases in the main field coefficients. We derived different models with different time intervals between \pm 240 and \pm 500 days and we found that variations in the internal Gauss coefficients (maximum of \pm 0.5 nT for $g_{1}^{0}$ ) indeed pointed at a problem of spectral leakage between external and internal field Gauss coefficients. The care taken in decimating the data on the quasi-regular grid, allowing for better separation in space between internal and static external fields, is not sufficient because the annual, semi-annual, and seasonal external field variations require year multiples to fully average out. Considering such multiples would have led us to choose a window width of \pm 182.5 days or \pm 365 days around 2005.0. According to Fig. 3, the conditioning number for the first window is poor, while according to Fig. 1, the second window would have led to some smoothing of the internal magnetic field time varia-

Table 1. Number $N$ of data points, mean of residuals, and rms misfit in nT for our parent model centered on epoch 2005.0. $B_{r}, B_{\theta}$ and $B_{\phi}$ are the three components of the magnetic field in a geocentric reference frame, with $r$ the radius, $\theta$ the co-latitude and $\phi$ the longitude.

\begin{tabular}{lccr}
\hline & $N$ & rms & Mean \\
\hline$F$ all & 26785 & 5.3 & -0.4 \\
$F$ lat $<\left|50^{\circ}\right|$ & 13087 & 4.1 & 0.1 \\
$F$ lat $>\left|50^{\circ}\right|$ & 13698 & 6.3 & -1.1 \\
$B_{r}$ & 11098 & 4.4 & -0.3 \\
$B_{\theta}$ & 11098 & 5.3 & -0.6 \\
$B_{\phi}$ & 11098 & 3.6 & 0.2 \\
\hline
\end{tabular}




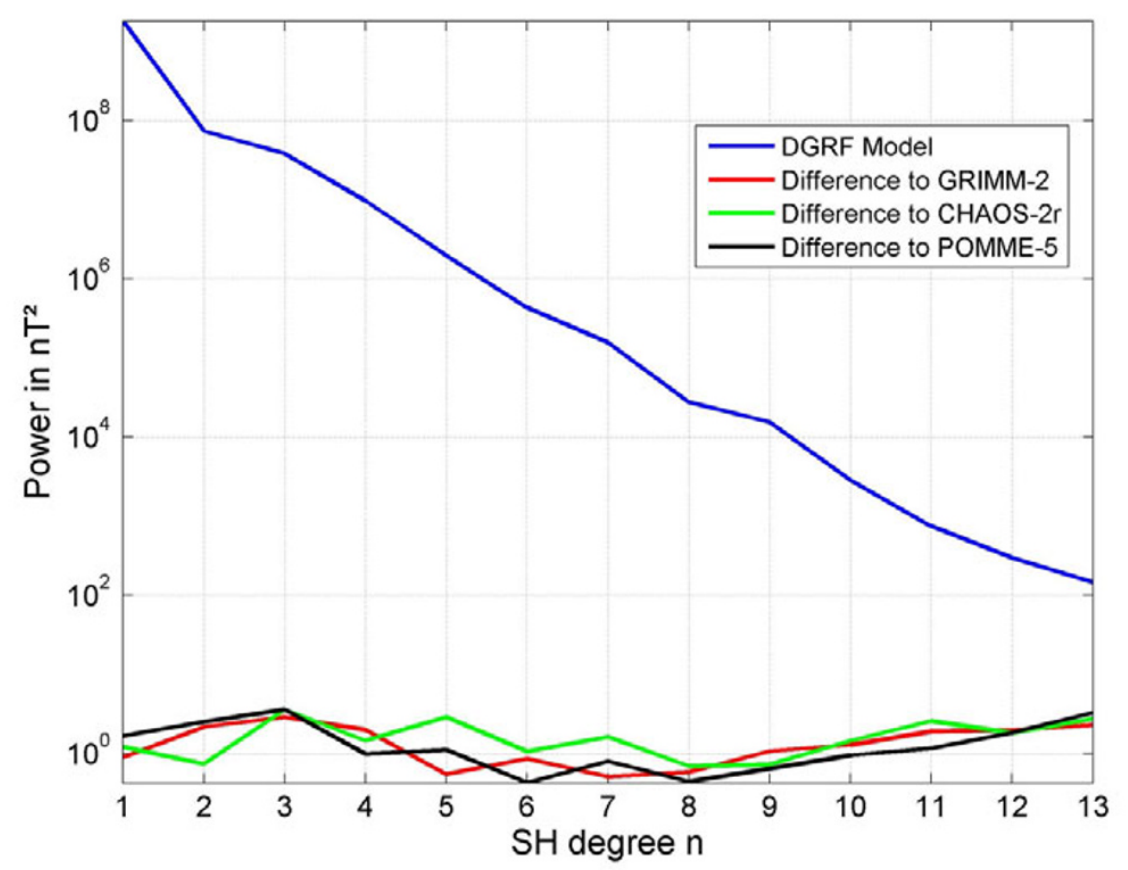

Fig. 4. Power spectrum of our parent 2005.0 candidate model (DGRF) at the Earth's mean radius. Difference between our model and others recently published at epoch 2005.0.

tion and thus to its underestimations. We faced here a contradiction that could hardly be resolved without resorting to a more sophisticated parameterization in time.

However, the error stemming from the external field leakage is about $\pm 0.5 \mathrm{nT}$ on MF Gauss coefficients and has low practical relevance. It should be compared to the rms due to truncating the MF model to SH degree 13 (and rounding the coefficients to two digits as was requested in the call for candidates) for generating the final 2005.0 candidate model, which is about $8 \mathrm{nT}$ (compared to the same model expanded to SH degree 15).

\section{2010 Main Field and Secular Variation Candi- date Models}

We followed the same approach for building the 2010 Main Field and Secular Variation candidate models but selected CHAMP satellite data centered on 2009.0 and up to 12th August 2009. The width of the time window is now 223 days and remains optimal if we consider the condition number (see Fig. 3). In the following, we call these models MF-09, SV-09, and SA-09, respectively for the main field, the secular variation and acceleration. The time window was large enough to obtain a good conditioning of the inverse problem but small enough to avoid the jerk seen at some magnetic observatories between 2006 and 2008 (Chulliat et al., 2010). In addition, we benefited from very quiet external field conditions. The number of scalar and vector data points, the rms and mean values of the residuals are listed in Table 2. Overall, there is a slight improvement in the statistics compared to epoch 2005 due to both the declining solar cycle and the smaller acceleration change in 2010 .
Table 2. Number $N$ of data points, mean of residuals, and rms misfit in nT for our parent model centered on epoch 2009.

\begin{tabular}{lccc}
\hline & $N$ & rms & Mean \\
\hline$F$ all & 32919 & 5.1 & -0.1 \\
$F$ lat $<\left|50^{\circ}\right|$ & 19085 & 4.1 & 0 \\
$F$ lat $>\left|50^{\circ}\right|$ & 13384 & 6.3 & -0.2 \\
$B_{r}$ & 17847 & 4.4 & 0.3 \\
$B_{\theta}$ & 17847 & 4.9 & -0.3 \\
$B_{\phi}$ & 17847 & 3.4 & 0.1 \\
\hline
\end{tabular}

\subsection{Assessment of the 2009.0 main field model}

Figure 5 shows the geographic distribution of intensity residuals between the selected CHAMP data and the MF-09 model. For this assessment, all CHAMP data belonging to the selected time interval were considered, not only those decimated on the quasi-regular grid. Figure 5 also shows the intensity residuals with respect to an independent Ørsted scalar dataset within the same time interval. The data underwent the same kind of selection and correction described above (see also Maus et al., 2010, this issue) but from which a lithospheric field model for degrees 14 to 40 was further removed.

CHAMP scalar residuals $\delta F$ have a complex structure, especially in the polar caps. Some of these small-scale structures at mid-latitudes are caused by the lithospheric field. However, most of the residuals are confined at high latitudes. The diamagnetic effect can be identified as a depression in the $\delta F$ residuals around the equator when premidnight data are examined (not shown here) but this latter effect is about $0.5 \mathrm{nT}$ on average.

Ørsted scalar residuals are different and display a signif- 

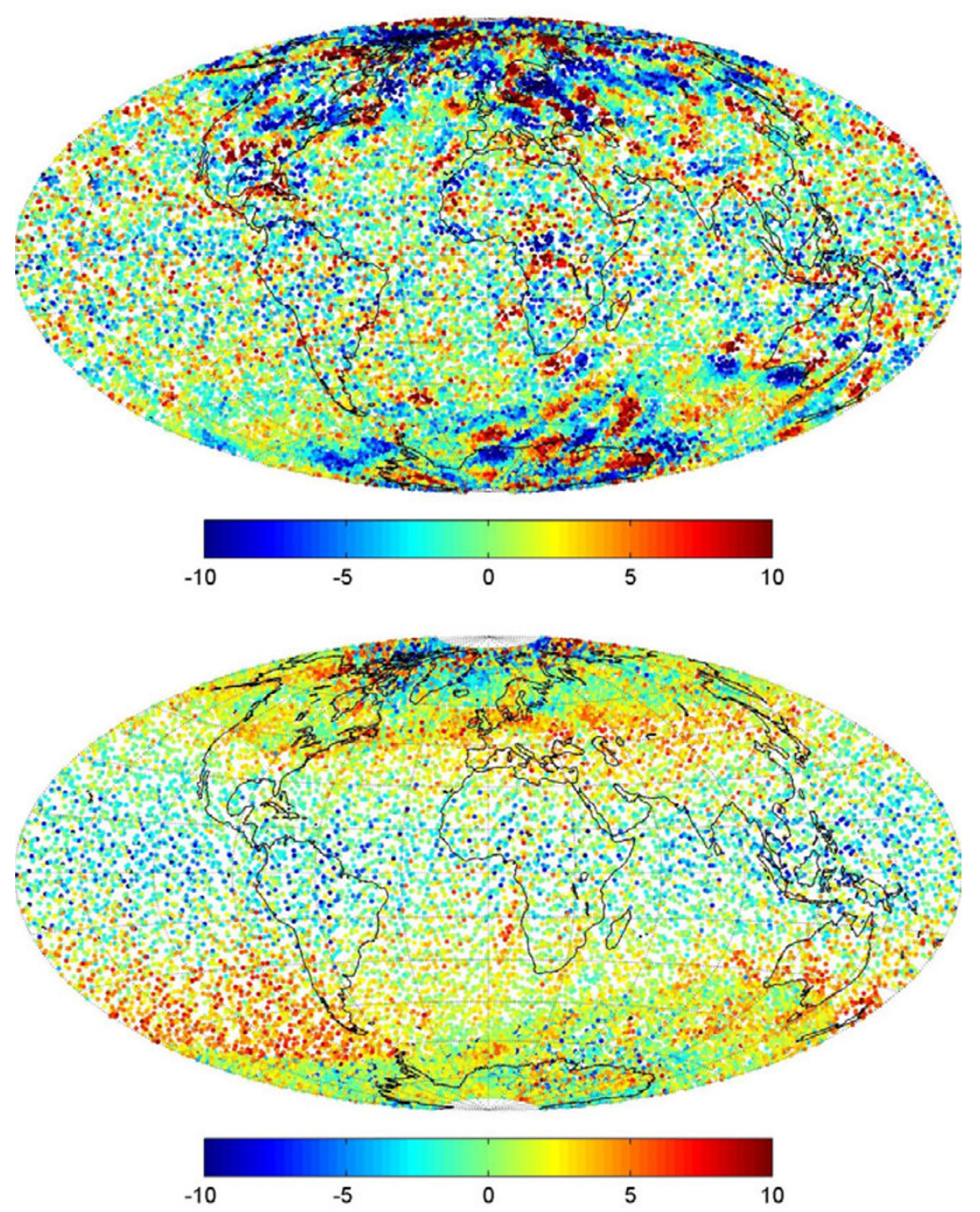

Fig. 5. Misfit between our model (MF09/SV09/SA09) and CHAMP scalar data (top); and Ørsted scalar data (bottom). Mollweide projection.

icant large-scale structure. Despite a low rms of $4.3 \mathrm{nT}$, the histogram of residuals shows a positive bias of $1.4 \mathrm{nT}$. Our model predictions thus underestimate the magnetic field contribution when evaluated at the altitude of Ørsted, particularly in the equatorial region. This initially led us to think that our model was contaminated by some diamagnetic effect. However, the same discrepancy was observed when Ørsted data were compared with another IGRF candidate model based on data corrected for the diamagnetic correction (Maus et al., 2010, this issue). In addition, the CHAOS-2 model that uses different parameterizations and datasets also shows suspicious mean residuals, positive for Ørsted and negative for CHAMP (see table 1 of Olsen et al., 2009). The incompatibility is not fully understood but it could originate from the calibration (see Maus et al., 2005a). Maus et al. (2010, this issue, figure 3) recently suggested that this could be the consequence of some baseline shift of one of the scalar CHAMP or Ørsted magnetometers after 2003. However, they could not infer from the data themselves whether the instrumental bias was coming from CHAMP or from Ørsted. A joint analysis of the synchro- nized measurements from vector and scalar magnetometers of onboard the two satellites would be needed to address this question. Our model performs apparently well but the apparent mismatch between CHAMP and Ørsted scalar data does not allow us to consider this test as fully conclusive.

The CHAOS-2r and -2s models could be used for comparisons with our models. We extracted from the CHAOS-2 models the Gauss coefficients, as well as their first time derivatives, for epoch 2009.0 and compared them to MF-09 and SV-09, respectively. Figure 6 shows the power spectra of the main field models, their secular variation, and their differences. The power of their differences for the main field distributes rather equally over all SH degrees. Despite a slight increase in the difference for $n=5$, we may reasonably conclude that there is no significant systematic error. The rms of the differences are $5.3 \mathrm{nT}$ and $4.3 \mathrm{nT}$ between MF-09 and the main fields of CHAOS-2s and CHAOS-2r, respectively. For the secular variation, we obtain $7.2 \mathrm{nT} / \mathrm{yr}$ and $5.6 \mathrm{nT} / \mathrm{yr}$ from the power spectra of the model difference. As expected, our non-regularized MF-09 model is in better agreement with the rough version of CHAOS-2. 


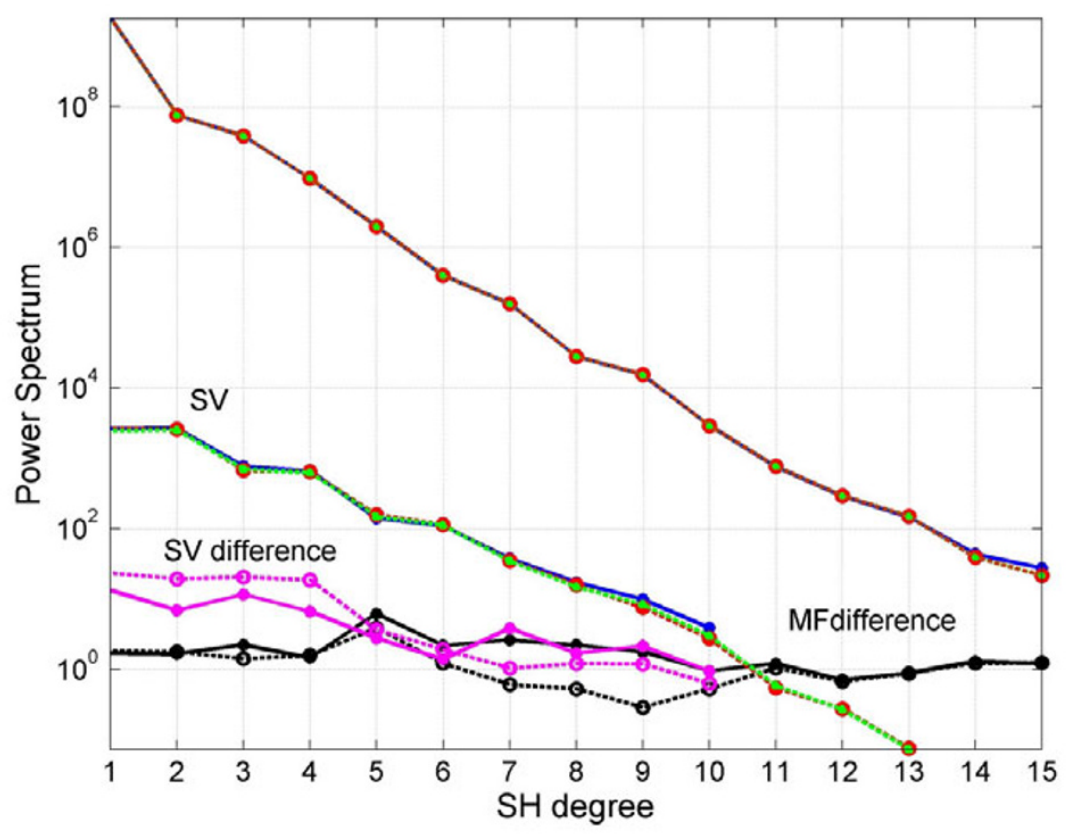

Fig. 6. Power spectra of our MF-09 and SV-09 models (blue), CHAOS-2s (red) and CHAOS-2r (green) for the main field in nT ${ }^{2}$ and the secular variation in $\mathrm{nT}^{2} / \mathrm{yr}^{2}$ for epoch 2009.0. The power spectra differences between our models and CHAOS-2r,s are shown in black for the main field and in pink for the secular variation. Solid line is the difference with respect to CHAOS-2s and the dashed line the difference with respect to CHAOS-2r.

\subsection{Assessment of the SV-09 model and the 2010.0 MF candidate}

Despite the different methodologies and datasets used to construct them, the SV-09 and SV-CHAOS-2r, and to a lesser extent the SV-CHAOS-2s, models are similar at epoch 2009.0 (Fig. 6). This provides some confidence that the SV-09 model is consistent enough for forecasting the main field MF-09 at epoch 2010.0. However, we carried out additional comparisons with independent data in the spatial domain.

Apart from Ørsted measurements, we have only a few independent data at our disposal to test the SV-09 model. Magnetic observatories usually distribute either definitive baseline-corrected data once a year or provisional data available within a delay of a few hours, but no intermediate baseline corrected data within a delay directly comparable to that of calibrated satellite measurements. Peltier and Chulliat (2010) recently proposed a method to bridge this gap and to produce quasi-definitive observatory data in a continuous fashion. We used their baseline-corrected data between November 2007 and October 2009 at nine observatories (AAE, BOX, CLF, KOU, LZH, MBO, PHU, PPT, TAM; the full names and positions of observatories may be found at www.intermagnet.org). Following, e.g., Mandea et al. (2000), we computed the differences between monthly means at $t+6$ months and $t-6$ months in order to remove seasonal variations in the observatory data (for instance, $\mathrm{SV}$ in June 2008 is computed from the monthly means in December 2008 and December 2007, only). These values, although possibly contaminated by geomagnetic activity of external origin, are likely to catch possible rapid internal field variations arising at one end of the interval. Figure 7 presents the SV time series for the three vector components at four of the selected observatories up to April 2009 (the latest date for which the SV could be computed from the quasi-definitive data) together with the SV-09 extrapolated with SA-09 to 2010. We selected BOX, CLF, TAM, and PPT because they sample very different geographical regions. The jerk of 2006-2008 is not of worldwide extent but is particularly prominent at mid-latitudes and on both sides of the Greenwich meridian (Chulliat et al., 2010). The SV in 2010 compares visually well with observatory quasidefinitive data at those various locations at the Earth's surface. Selecting data posterior to 2008 for deriving our models enables to capture the recent trend of the secular variation at the end of each observatory time series. Considering the nine observatories, the rms between model values and data in April 2009 are 10.4 nT/yr, 3.5 nT/yr, and $8.3 \mathrm{nT} / \mathrm{yr}$, respectively for $X, Y$, and $Z$. Interpreting these values on such a small number of observatories is subtle because the errors incurred in determining the time variations from the difference of monthly means may be large. The $X$ component, in particular, is known to be contaminated at mid-latitudes by magnetic fields generated by high-latitude currents during geomagnetically disturbed times, and at low latitudes by the equatorial electrojet.

We therefore also performed the same analysis on a statistically more significant number of samples composed of monthly means from 86 INTERMAGNET observatories available from January 2007 to December 2008 (definitive data). For each observatory, three estimates of the SV (SV-1, SV-2, and SV-3) were computed to challenge the robustness of our results. SV-1 was calculated as before (i.e. by taking the difference between monthly mean values at $t+6$ months and $t-6$ months); SV-2, a smoother estimate, was computed as the mean of the last four SV-1 values, and 

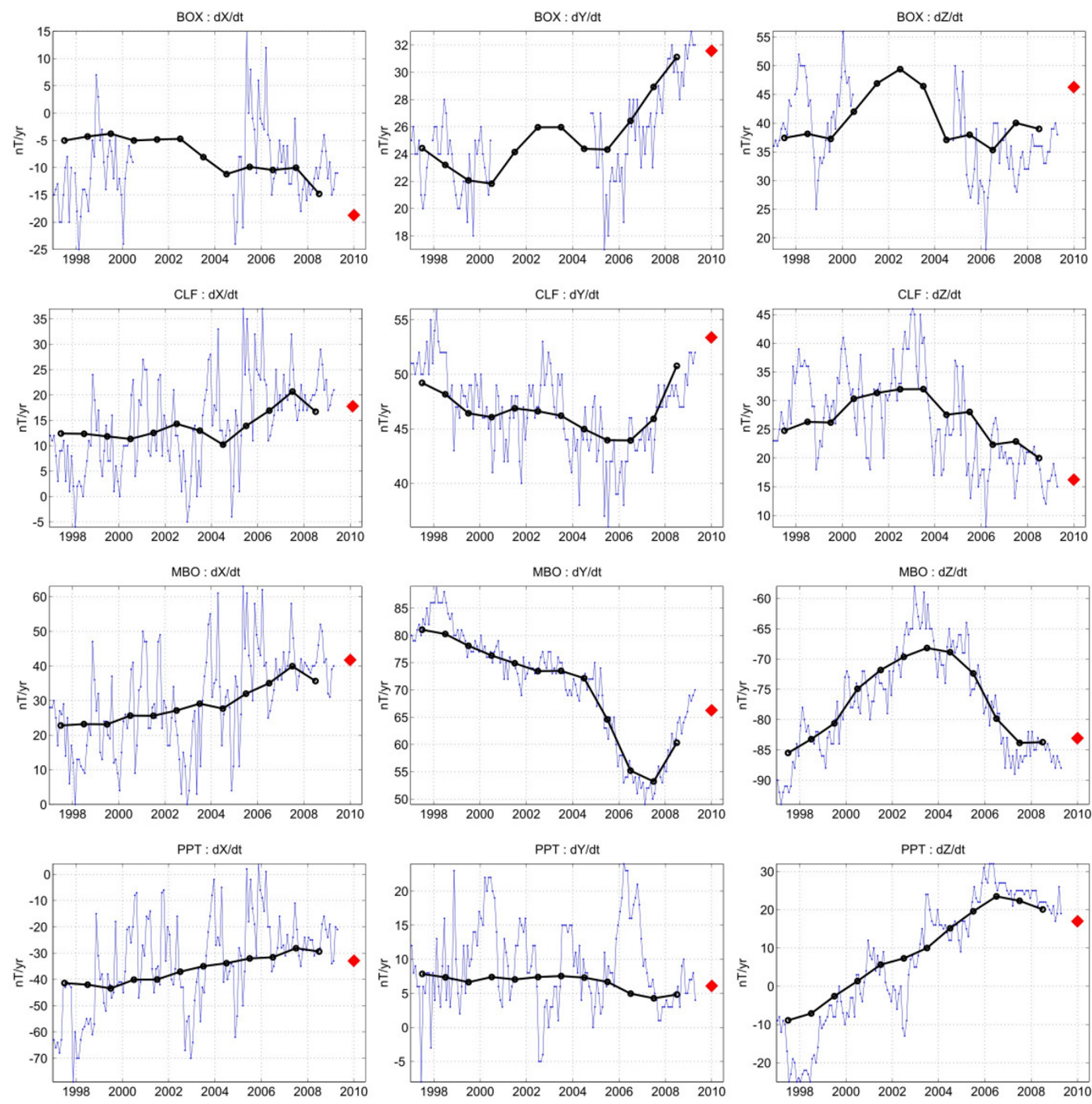

Fig. 7. Observatory definitive (prior to epoch 2009.0) and quasi-definitive (after 2009) monthly mean values for $d X / d t$ (left), $d Y / d t$ (middle), and $d Z / d t$ at BOX, CLF, MBO, and PPT observatories selected for their geographical location. Black circles are yearly mean values computed from CHAOS-2s model and the red diamonds indicate the SV predictions of our SV-09 model extrapolated to 2010.0 with the help of our SA-09 model.

$\mathrm{SV}-3$, an even smoother estimate, was computed from the last twelve SV-1 values.

Using these definitive INTERMAGNET observatory data, the latest SV evaluation recovered in this way is for June 2008. Figure 8 summarizes the residuals between the SV-09 (truncated to SH degree 8 as required for an IGRF candidate) and the SV estimates at observatories for the three different cases (SV-1, SV-2, and SV-3). Overall, the three ways of estimating the SV agree remarkably well. This is certainly a positive effect of the minimum solar activity over the period studied here. There is a slight bias in the horizontal components (about $-2.5 \mathrm{nT} / \mathrm{yr}$ for $X$ and $1.5 \mathrm{nT} / \mathrm{yr}$ for $Y$, for the three types of SV) but none on $Z$. The total bias is comparable to the effect of truncating the SV-09 to SH degree 8 (according to Fig. 6, the contribu- tions of SH degrees 9 and 10 are indeed about $3.7 \mathrm{nT} / \mathrm{yr}$ ). Moreover, despite a few isolated large residuals (an absolute maximum of $-17 \mathrm{nT} / \mathrm{yr}$ for $Z$ at PHU, Vietnam) the standard deviation of the distributions in Fig. 7 are between 3.5 and $5.5 \mathrm{nT} / \mathrm{yr}$ for all components. Such values are also consistent with the rms found in comparing SV-09 with SV-CHAOS-2r. Our truncated SV-09 thus appears to be consistent with an independent model and with both quasidefinitive and definitive observatory data. As a result, we used the SV-09 coefficients to linearly extrapolate MF-09 to epoch 2010.0 and generate our MF 2010 candidate model.

Note that a similar evaluation method based upon observatory data was applied to all candidate models by Chulliat and Thébault (2010, this issue) as part of the IGRF evaluation process. 

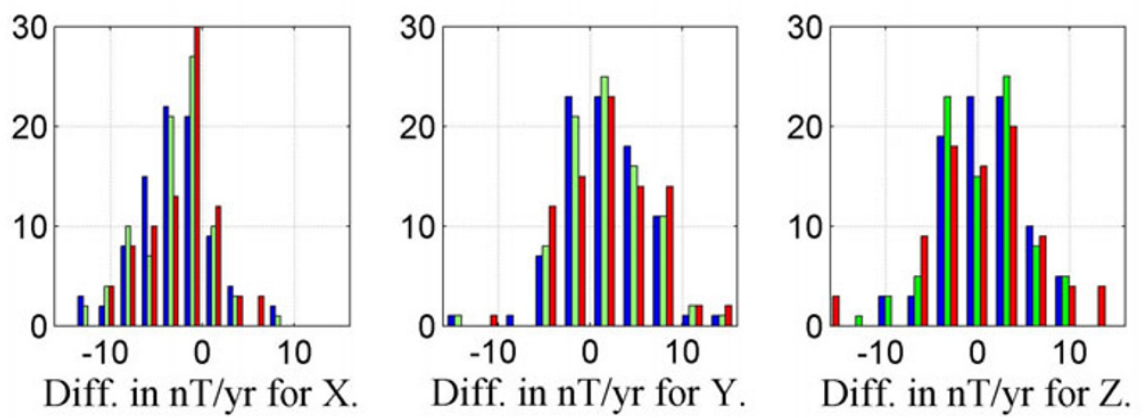

Fig. 8. Distribution of residuals for the three magnetic field components between SV-09 and the SV estimated at 86 INTERMAGNET observatories using the method SV-1 (blue), SV-2 (green), and SV-3 (red). See text for details.

\subsection{SV 2010-2015 candidate model}

To produce a SV 2010-2015 candidate model, we need to decide whether we should extrapolate the SV-09 model to post 2009.0 epochs (within the 2010-2015 time interval of IGRF-11). The answer is not trivial and again a matter of compromise. We have the choice between keeping an "instantaneous" estimate of the SV, and relying on the rule of thumb stating that 5 years SV forward prediction generally requires a SV model constructed with data covering a similar time range. By deriving our SV-09 from a rather short time span ( \pm 240 days) we implicitly chose the first option and assumed that our model has a good predictability over short time scales. Nevertheless, we note that extrapolating SV-09 to epochs posterior to 2009.0 by applying the coefficients of SA-09 is another option that also requires some consideration.

Such an option would need to assume that acceleration changes would be negligible in the near future. Chulliat et al. (2010) pointed out that rapid magnetic field acceleration changes occurred around 2007, following core field acceleration in 2005-2006. From 2007 until the end of 2009, the quasi-definitive observatory data suggest that the field acceleration did not bear noticeable changes at the selected observatories. We could thus have speculated that the acceleration will not vary much in the coming few years and that SA-09 can be used to extrapolate SV-09 to 2012.5, for instance. However, an analysis of the SA computed from the CHAOS-2r, to which our model is closer than to CHAOS-2s, led us to conclude that the secular acceleration coefficients determined from data spanning short time intervals are of dubious relevance for extrapolation. As noted earlier, the contamination of external fields is the main limiting factor when the acceleration coefficients are obtained from data that do not cover an interval of yearly multiples. Consequently, we decided not to take the risk of extrapolating the SV-09 model forward in time. SV-09 was thus considered as representative of the SV-2010-2015 and, after truncation to SH 8 and rounding to two digits, submitted as our SV candidate model to IGRF-11.

\section{Concluding Remarks}

We presented simple (not simplistic) candidate field models based on a quadratic time representation of the main magnetic field and on external field parameterized with magnetic indices derived from ground-based observatories. These models were derived from selected and pre-processed CHAMP satellite data. We showed that such an approach could lead to satisfying IGRF-11 candidate models if we accept some compromises and use information about the core field acceleration that is provided by independent data and models. Such compromises may be summarized as follows.

Our candidate model for epoch 2005.0 is a retrospective snapshot model of the main field at a fixed epoch derived from a set of geographically incomplete and noisy data. If we rejected the idea of introducing mathematical regularization in the inverse problem, we could not avoid adding some a priori information (thus implicit regularization) derived from other magnetic field models. The recent models, such as the CHAOS family, revealed an intense pulse of acceleration near 2005 (Chulliat et al., 2010). For the construction of our 2005.0 candidate model, we viewed this change of acceleration as the major magnetic field event and selected the data time interval accordingly. This choice implied choosing a narrow time window in order to justify neglecting the third time derivative in the Taylor expansion of Gauss coefficients. This was made at the cost of slightly degrading the representation of the annual external field variations. As a result, it may well be that the gain we supposedly acquired was at the expense of some Gauss coefficients, probably those corresponding to zonal terms (Olsen et al., 2005b). There was no satisfying trade-off for that epoch. It is widely known that the external field activity driven by the Sun is important for selecting the data but our study also shows that knowing if the core field is in a minimum, ascending, maximum, or descending phase of acceleration is equally instructive if we adopt a modeling scheme based on a simple parameterization in time.

The same information about the core field acceleration was necessary for deriving our model candidates for 2010.0. It convinced us to select data posterior to 2008 in order to ensure that the newly detected jerk in 2007 (Chulliat et al., 2010), which actually spans years 2006-2008 depending on the location of magnetic observatories, was not contained in the selected satellite data. A third order Taylor expansion of the internal Gauss coefficients would otherwise have been needed. In a sense, we only retained "quiet" times core field acceleration that, by chance, also corresponded to quiet external field conditions due to the solar minimum of 
activity. The selection of quiet acceleration conditions illustrates well why forecasting the main field with pure mathematical extrapolation remains a major challenge. It is indeed impossible to assert that our SV model obtained for quiet acceleration periods (not low acceleration but of negligible variation) will represent well near-future time evolutions of the core field. We could have followed the common practice that consists in deriving SV candidate models with data spanning about five years (see table 1 of Maus et al., 2005b). However, with the help of Fig. 1 one can see that this procedure, by smoothing the different phases of core field acceleration, mitigates the risk of introducing too steep MF time variations but does not better address the issue raised by the model predictability over the next 5-year.

For all candidate models, we are generally missing a true error estimate. Formal errors on Gauss coefficients, calculated from the covariance matrix and the a posteriori estimated data error, are not helpful to assess the true accuracy of our model with respect to an arbitrary and independent dataset. The magnetic field inverse problem was implicitly regularized by setting the maximum $\mathrm{SH}$ degree, selecting the quadratic time basis functions, and ensuring that the chosen time window and the data distribution were compatible with these settings. Our Gauss coefficients were thus artificially well-determined (recall Fig. 3) and the error estimations mostly driven by the data error. Unfortunately, the estimated data error itself is misleading because it largely reflects the quality of the selection criteria and the correction applied to the data before the inversion. Figure 1 shows that the mathematically estimated error of coefficient $\ddot{g}_{1}^{1}$ does not contain the estimates of the CHAOS- 2 model. The size of the error bars indicates the low level of freedom of our coefficient with respect to the entire model parameterization, not with respect to an arbitrary set of independent magnetic data. We thus had to challenge our models with other available independent data in order to estimate their error. However, we must keep in mind that such independent data also underwent some kind of selection criteria.

The major problem in our models is not so much the intrinsic data error but the pernicious leakage of external field into internal geomagnetic field models that introduces some bias (see also Olsen et al., 2005b). This problem is typical of models based on measurements made by single satellite missions that cannot provide measurements desynchronized in space and time. Addressing this issue is the purpose of the forthcoming SWARM mission that will include for the first time a constellation of three satellites on different orbits (Friis-Christensen et al., 2006). The 12th generation of IGRF (planned for 2015) will thus certainly out-date most of the problems discussed in this paper. The new mission will prompt innovative ways of representing the Earth's magnetic field, either mathematically, or physically by incorporating dynamical constraints in the general framework of data assimilation (e.g. Fournier et al., 2007), but the improved sampling in space and time of SWARM measurements will also greatly enhance the robustness of simpler approaches such as the one presented here.
Acknowledgments. The German CHAMP satellite is operated by GeoForschungsZentrum Potsdam, supported by the German Aerospace Center (DLR) and the Federal Ministry of Education and Research (BMBF). The Ørsted Project was made possible by extensive support from the Danish Government, NASA, ESA, CNES and DARA. We thank the national institutes that support magnetic observatories and INTERMAGNET for promoting high standards of magnetic observatory practice (www.intermagnet.org). We would like to thank N. Gillet and M. Hamoudi for their thorough review. The work was supported by CNES. For IPGP this is contribution number 2650.

\section{References}

Chulliat, A. and E. Thébault, Testing IGRF-11 candidate models against CHAMP data and quasi-definitive observatory data, Earth Planets Space, 62, this issue, 805-814, 2010.

Chulliat, A., E. Thébault, and G. Hulot, Core field acceleration pulse as a common cause of the 2003 and 2007 geomagnetic jerks, Geophys. Res. Lett., 37, L07301, doi:10.1029/2009GL042019, 2010.

Fournier, A., C. Eymin, and T. Alboussière, A case for variational geomagnetic data assimilation: insights from a one-dimensional, nonlinear, and sparsely observed MHD system, Nonlin. Proc. Geophys., 14(2), 163180, 2007.

Friis-Christensen, E., H. Lühr, and G. Hulot, Swarm: A constellation to study the Earth's magnetic field, Earth Planets Space, 58(4), 351-358, 2006.

Hamoudi, M., E. Thébault, V. Lesur, and M. Mandea, GeoForschungsZentrum Anomaly Magnetic Map (GAMMA): A candidate model for the World Digital Magnetic Anomaly Map, Geochem. Geophys. Geosyst., 8, Q06023, doi:10.1029/2007GC001638, 2007.

Hemant, K., E. Thébault, M. Mandea, D. Ravat, and S. Maus, Magnetic anomaly map of the world: merging satellite, airborne, marine and ground-based magnetic data sets, Earth Planet. Sci. Lett., doi:10.1016/j.eps1.2007.05.040, 2007.

Korhonen, J., D. Fairhead, M. Hamoudi, K. Hemant, V. Lesur, M. Mandea, S. Maus, M. Purucker, D. Ravat, T. Sazonova, and E. Thébault, Magnetic Anomalie Map of the World / Carte des anomalies magnétiques $d u$ monde, 1st edition, 1:50,000,000,CCGM/CCGMW, ISBN 978-952217-000-2, 2007.

Kuvshinov, A. and N. Olsen, 3-D modelling of the magnetic fields due to ocean tidal flow, in CHAMP Mission Results II, 359-365, Springer Berlin, 2004.

Lesur, V., I. Wardinski, M. Rother, and M. Mandea, GRIMM: the GFZ Reference Internal Magnetic Model based on vector satellite and observatory data, Geophys. J. Int., 173(2), 382-394, 2008.

Lühr, H., M. Rother, S. Maus, W. Mai, and D. Cooke, The diamagnetic effect of the equatorial Appleton anomaly: Its characteristics and impact on geomagnetic field modeling, Geophys. Res. Lett., 30(17), 1906, doi:10.1029/2003GL017407, 2003.

Macmillan, S. and S. Maus, International Geomagnetic Reference Fieldthe tenth generation, Earth Planets Space, 57, 1135-1140, 2005.

Mandea, M., E. Bellanger, and J.-L. Le Mouël, A geomagnetic jerk for the end of the 20th century?, Earth Planet. Sci. Lett., 183, 369-373, 2000.

Maus, S. and P. Weidelt, Separating the magnetospheric disturbance magnetic field into external and transient internal contributions using a 1D conductivity model of the Earth, Geophys. Res. Lett., 31(12), L12614, 10.1029/2004GL020232, 2004.

Maus, S., S. McLean, D. Dater, H. Lühr, M. Rother, W. Mai, and S. Choi, NGDC/GFZ candidate models for the 10th generation of IGRF, Earth Planets Space, 57, 1151-1156, 2005a

Maus, S., S. Macmillan, F. Lowes, and T. Bondar, Evaluation of candidate geomagnetic field models for the 10th generation of IGRF, Earth Planets Space, 57, 1173-1181, 2005b.

Maus, S., M. Rother, C. Stolle, W. Mai, S. Choi, H. Lühr, D. Cooke, and C. Roth, Third generation of the Potsdam Magnetic Model of the Earth (POMME), Geochem. Geophys. Geosyst., 7, Q07008, doi:10. 1029/2006GC001269, 2006.

Maus, S., T. Sazonova, K. Hemant, J. D. Fairhead, and D. Ravat, National Geophysical Data Center candidate for the World Digital Magnetic Anomaly Map, Geochem. Geophys. Geosyst., 8, Q06017, doi:10. 1029/2007GC001643, 2007.

Maus, S., C. Manoj, J. Rauberg, I. Michaelis, and H. Lühr, NOAA/NGDC candidate models for the 11th generation International Geomagnetic Reference Field and the concurrent release of the 6th generation Pomme 
magnetic model, Earth Planets Space, 62, this issue, 729-735, 2010.

Menvielle, M. and A. Marchaudon, Geomagnetic indices in solarterrestrial physics and space weather, in Space Weather, edited by J. Lilensten, 277-288, Springer, 2006.

Olsen, N. and M. Mandea, Investigation of a secular variation impulse using satellite data: The 2003 geomagnetic jerk, Earth Planet. Sci. Lett., 255, 94-105, 2007.

Olsen, N., R. Holme, G. Hulot, T. J. Sabaka, T. Neubert, L. ToffnerClausen, F. Primdahl, J. Jorgensen, J. M. Leger, D. Barraclough, J. Bloxham, J. Cain, C. Constable, V. Golovkov, A. Jackson, P. Kotze, B. Langlais, S. Macmillan, M. Mandea, J. Merayo, L. Newitt, M. Purucker, T. Risbo, M. Stampe, A. Thomson, and C. Voorhies, Ørsted initial field model, Geophys. Res. Lett., 27, 3607-3610, 2000.

Olsen, N., T. J. Sabaka, and F. Lowes, New parameterization of external and induced fields in geomagnetic field modeling, and a candidate model for IGRF 2005, Earth Planets Space, 57, 1141-1149, 2005a.

Olsen, N., F. Lowes, and T. J. Sabaka, Ionospheric and induced field leakage in geomagnetic field models, and derivation of candidate models for DGRF 1995 and DGRF 2000, Earth Planets Space, 57, 1191-1196,
$2005 b$.

Olsen, N., M. Mandea, T. J. Sabaka, and L. Tøffner-Clausen, CHAOS2 a geomagnetic field model derived from one decade of continuous satellite data, Geophys. J. Int., 179, 1477-1487, doi: 10.1111/j.1365246X.2009.04386.x, 2009.

Peltier, A. and A. Chulliat, On the feasibility of promptly producing quasidefinitive magnetic observatory data, Earth Planets Space, 62, e5-e8, doi:10.5047/eps.2010.02.002, 2010.

Reigber, C., H. Lühr, and P. Schwintzer, CHAMP mission status, Adv. Space Res., 30, doi:10.1016/S0273-1177(02)00276-4, 2002.

Thébault, E., Global lithospheric magnetic field modeling by successive regional analysis, Earth Planets Space, 58, 485-495, 2006.

Thomson, A. W. P. and V. Lesur, An improved geomagnetic data selection algorithm for global geomagnetic field modelling, Geophys. J. Int., 169(3), 951-963, 10.1111/j.1365-246X.2007.03354.x, 2007.

E. Thébault (e-mail: ethebault@ipgp.fr), A. Chulliat, S. Maus, G. Hulot, B. Langlais, A. Chambodut, and M. Menvielle 